\title{
A 45-MHz continuum survey of the northern hemisphere
}

\author{
K. Maeda ${ }^{1}$, H. Alvarez ${ }^{2}$, J. Aparici ${ }^{2}$, J. May ${ }^{2}$, and P. Reich ${ }^{3}$ \\ 1 Department of Physics, Hyogo College of Medicine, Nishinomiya, 663-8501, Japan \\ 2 Departamento de Astronomía, Universidad de Chile, Casilla 36-D, Santiago, Chile \\ 3 Max-Plank-Institut für Radioastronomie, Auf dem Hügel 69, 53121 Bonn, Germany
}

Received June 4; accepted September 3, 1999

\begin{abstract}
We present a survey of the $45 \mathrm{MHz}$ radio continuum emission in the declination range $+5^{\circ}$ to $+65^{\circ}$. The observations were made with the circular filled array of the Japanese Midle and Upper Atmosphere Radar with a half-power beam width of 3.6 . The results are presented in sets of maps in galactic and equatorial coordinates (Epoch $1950)$.
\end{abstract}

Key words: radio continuum: ISM — surveys — galaxy: structure

\section{Introduction}

Since the beginning of radio astronomy several surveys of the galactic background have been made at various frequencies, but most of them covered only the limited region near the galactic plane (see e.g., Salter \& Brown 1988).

It is presently known that the background continuum radiation is a mixture of thermal free-free emission from ionized hydrogen and non-thermal synchrotron emission resulting from the interaction of cosmic ray electrons with the galactic magnetic field. At frequencies higher than about $1 \mathrm{GHz}$, the diffuse thermal component becomes important in the galactic plane; for example, at $1420 \mathrm{MHz}$ it may reach $30 \%$ to $50 \%$ (Reich 1986). The non-thermal component is important at low frequencies and increases with decreasing frequency until the free-free absorption due to ionized hydrogen sets in at around a few tens of $\mathrm{MHz}$. Continuum surveys in this range (say $20 \mathrm{MHz} \leq \nu \leq$ $100 \mathrm{MHz}$ ) are therefore most important to investigate the structure of the magnetic field in our Galaxy, the spatial and energy distributions of cosmic ray electrons as well as those of the synchrotron radio emission. However, only a few surveys, discussed in Sect. 5, have been made in this frequency range because of inherent difficulties. For

Send offprint requests to: H. Alvarez example, to achieve a good angular resolution at low frequencies it is necessary to build a large array; terrestrial interference (man-made and natural) generally becomes serious with decreasing frequency; the ionospheric opacity increases as the frequency decreases, and changes in opacity result in spurious intensity variations of the incoming background radiation. Furthermore, solar radio bursts fatally disturb the observation of the background radiation during high solar activity periods.

In this paper we present the results of a northern survey at $45 \mathrm{MHz}$. The observations were made with the MU (Middle and Upper atmosphere) radar located at Shigaraki, Japan. The data processing was performed at the Maipu Radio Observatory, Chile, and the final maps were obtained at the Max-Planck-Institut für Radioastronomie, Germany, making use of the NOD2 program package (Haslam 1974).

\section{Observations}

\subsection{The $M U$ radar array}

The MU radar is primarily used for Doppler shift measurements of the echo signals which are incoherently scattered by the atmospheric particles at various heights. Since the detailed description of the MU radar has been given elsewhere (Fukao et al. 1985a, 1985b), here we will only briefly describe the system which is an active-phased array consisting of 475 crossed 3-element Yagis arranged within a circle of $103-\mathrm{m}$ diameter. It has 475 transmitter-receiver modules, each connected to crossed Yagis by two equallength coaxial cables. By phasing the two orthogonal Yagis by means of a phase switch, the right-hand or left-hand polarization are obtained. Each transmitter-receiver module has a preamplifier and a phase shifter. There is only one beam and, by electronically controlling the phasing parameters, its direction can be changed from one position to the next, as rapidly as $0.4 \mathrm{~ms}$. The Yagi elements are not physically tilted as the array is phased to various 
Table 1. Parameters of the MU radar

\begin{tabular}{ll}
\hline Operating frequency & $46.5 \mathrm{MHz}$ \\
Array & $475 \mathrm{crossed}$ 3-element Yagis \\
Half-power beam width & $3.6(\alpha) \times 3.6 \mathrm{sec} Z(\delta)$ \\
Polarization & circular $(\mathrm{RH}$ or $\mathrm{LH})$ or linear \\
Effective area & $8300 \mathrm{~m}^{2}$ at the zenith \\
Beam direction & $0^{\circ} \leq Z^{\mathrm{a}} \leq 30^{\circ} ; 0^{\circ} \leq A^{\mathrm{b}} \leq 360^{\circ}$ \\
Receiver bandwidth & $1.65 \mathrm{MHz}$ \\
Array coordinates & $34.85 \mathrm{~N}, 136.10 \mathrm{E}$
\end{tabular}

a Zenith angle.

b Azimuth.

zenith angles. The beam direction can be tilted as far as $30^{\circ}$ from the zenith. A basic triangular grid is used to arrange the array elements. The element spacing is $0.7 \lambda$ and no significant grating lobes appear for $Z \leq 30^{\circ}$, where $Z$ is the zenith angle. The first side lobe level is theoretically $-18 \mathrm{~dB}$ with respect to the main beam. The maximum effective area of the array is $8300 \mathrm{~m}^{2}$ and the half-power beam width is 3.6 at the zenith. As $Z$ increases, the effective area decreases in proportion to $\cos Z$ because of foreshortening and the beam within a tipped direction also broadens as $3.6 \sec Z$. Since the beam direction of the MU radar can be tilted up to $30^{\circ}$ from the zenith, we can observe the sky in the declination range from +4.85 to +64.85 , and the reduction in effective area is less than $15 \%$. The array polarization is almost purely circular in every direction at least for $Z \leq 30^{\circ}$.

Since the 475 preamplifiers are simultaneously working during observation, it is practically impossible to make calibrations. It is therefore necessary to calibrate the MU radar observations using calibrated independent data. The system noise is about $2300 \mathrm{~K}$. Table 1 shows the main instrumental parameters of the MU radar array.

\subsection{Survey observations}

Our observations were made in the periods 1985-1989 and 1997-1999, each consisting of 2-4 day runs. Since some of the observations experienced interference and apparent gain changes, we used only selected data for this survey. The results presented in this paper are based on the data taken during February 24-26, 1988, and February 9-11, 1998. Data sets taken on other occasions were used as a complement in the analysis.

In the observations, the MU radar was used as a meridian transit instrument by taking advantage of the quick steerability of the beam direction. For each one-day observation a total of 16 or 18 declinations were observed. The beam direction was changed every $1 \mathrm{~ms}$ and the selected declinations were cyclically scanned. The declinations observed in 1988 (two different sets of 16 beam directions) and 1998 (one set of 18 beam directions) runs are shown
Table 2. Observed declination strips in 1988 and 1998

\begin{tabular}{|c|c|c|c|}
\hline $\begin{array}{l}\delta(1988) \\
\left(^{\circ}\right)\end{array}$ & $\begin{array}{l}\delta(1998) \\
\left({ }^{\circ}\right)\end{array}$ & $\begin{array}{l}\delta(1988) \\
\left({ }^{\circ}\right)\end{array}$ & $\begin{array}{l}\delta(1998) \\
\left(^{\circ}\right)\end{array}$ \\
\hline 4.85 & 4.85 & 34.85 & 34.85 \\
\hline - & 6.85 & 36.85 & - \\
\hline 8.85 & - & - & 38.85 \\
\hline- & 10.85 & 40.85 & 40.85 \\
\hline 12.85 & 12.85 & - & 42.85 \\
\hline- & 14.85 & 44.85 & - \\
\hline 16.85 & - & - & 46.85 \\
\hline- & 18.85 & 48.85 & - \\
\hline 20.85 & - & - & 50.85 \\
\hline 21.85 & - & 52.85 & - \\
\hline - & 22.85 & - & 54.85 \\
\hline 24.85 & - & 56.85 & - \\
\hline- & 26.85 & 58.85 & 58.85 \\
\hline 28.85 & - & 60.85 & - \\
\hline- & 30.85 & - & 62.85 \\
\hline 32.85 & - & 64.85 & - \\
\hline
\end{tabular}

in Table 2. We have not used all the declinations of the 1998 data, but only those which have not been observed in 1988.

\section{Data reduction}

The pointing accuracy was checked using the raw data of the four strong radio sources Cas A, Cyg A, Tau A, and Vir A. The center of the drift curve of each source was estimated and compared with the corresponding source position. Deviations smaller than 0.2 , and to the east, were found for each of these sources. It was assumed that all the other pointings were also located along the local meridian plane with the same accuracy.

Since the MU radar has no calibration system we used the Maipu 45-MHz survey of the University of Chile (Alvarez et al. 1997) to calibrate the MU radar observations. The MU-radar and the Maipu arrays have important similar characteristics: the Chilean array, consisting of 528 full-wavelength dipoles, has an effective area of $11200 \mathrm{~m}^{2}$, comparable to that of the Japanese array; the operating frequency of the Chilean array $(45 \mathrm{MHz})$ is very close to that of the MU radar $(46.5 \mathrm{MHz})$, and the beam width of the Maipu array is $4.6 \times 2.4$, also close to that of the MU radar (3.6). Since the declination coverage of the southern array is $-86^{\circ}$ to $+19^{\circ}$, the instruments can observe a common declination range $+5^{\circ}$ to $+19^{\circ}$.

The data were smoothed by software simulating a normal RC filter in a detector. The integrating process was performed in direct and reversed time to minimize time shifting. The time constant of the filter was $60 \mathrm{~s}$. Previous to this step, cleaning and excising of static bursts or interference was required in some of the observations. 


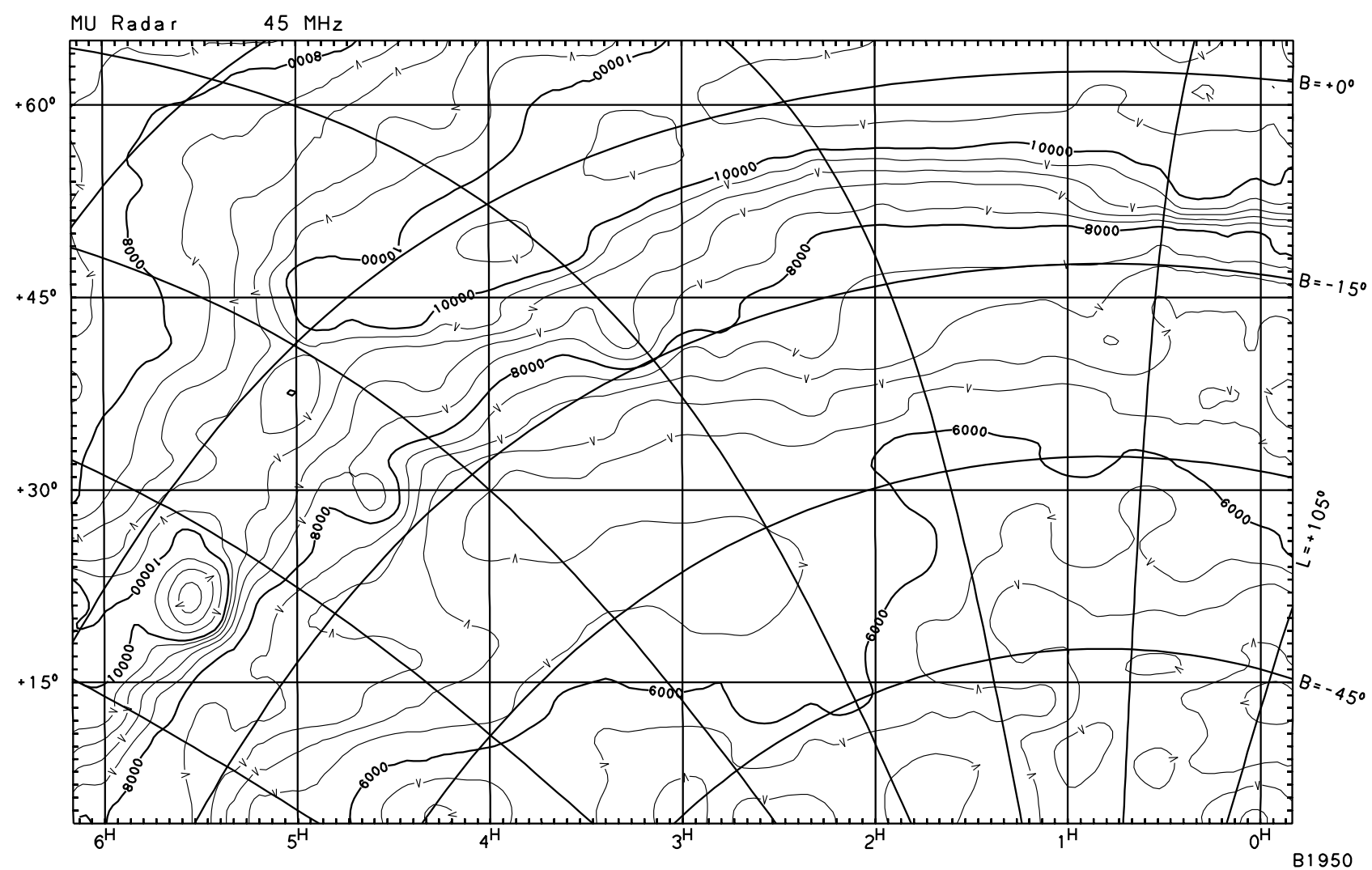

Fig. 1. Set of maps presented in equatorial coordinates (Epoch 1950). Each panel covers 6 hours in right ascension, and $61^{\circ}$ $\left(+4^{\circ} \leq \delta \leq+65^{\circ}\right)$ in declination. Contours are labelled in Kelvin (see text). Arrows on contour lines point towards decreasing temperatures

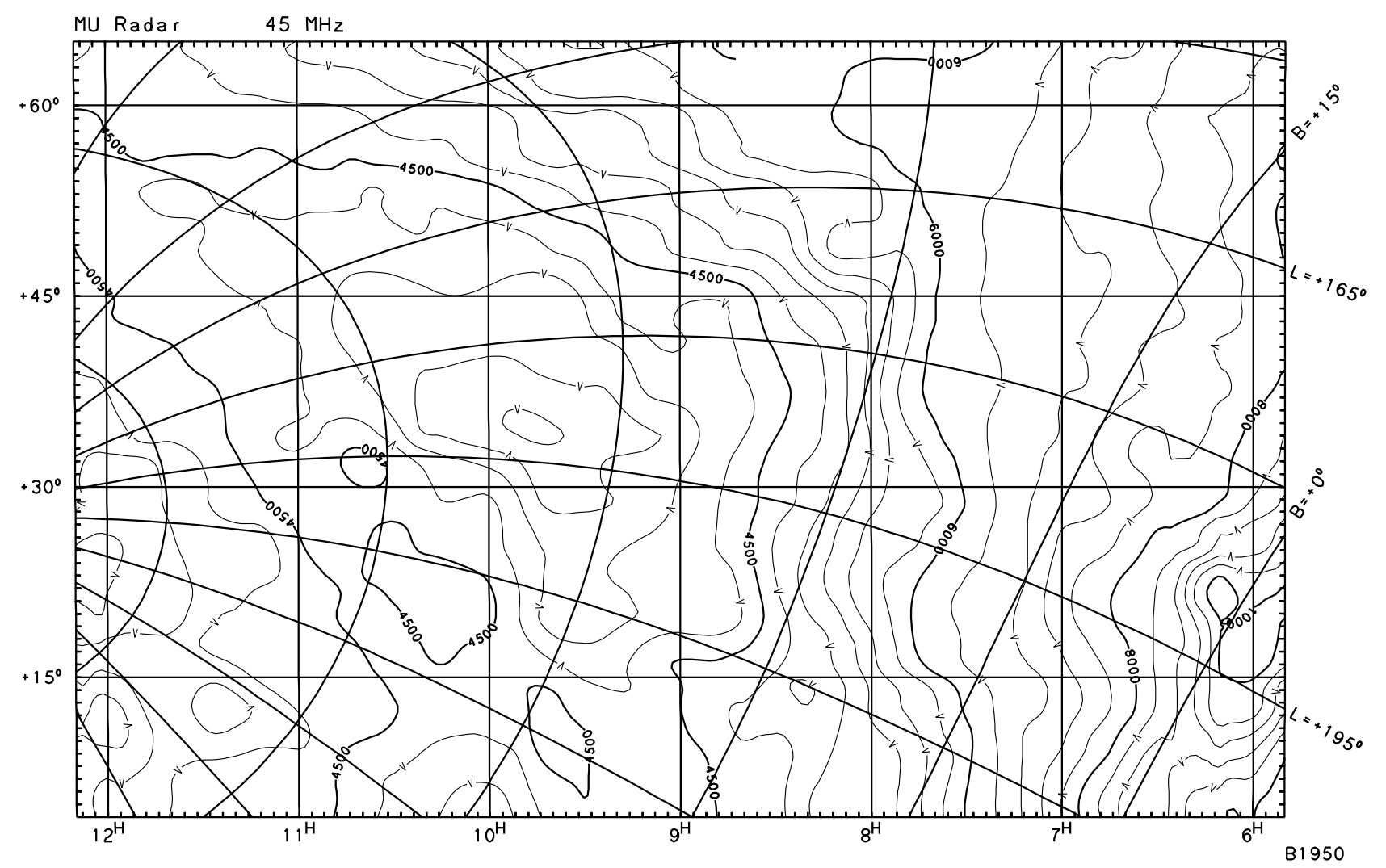

Fig. 1. continued 


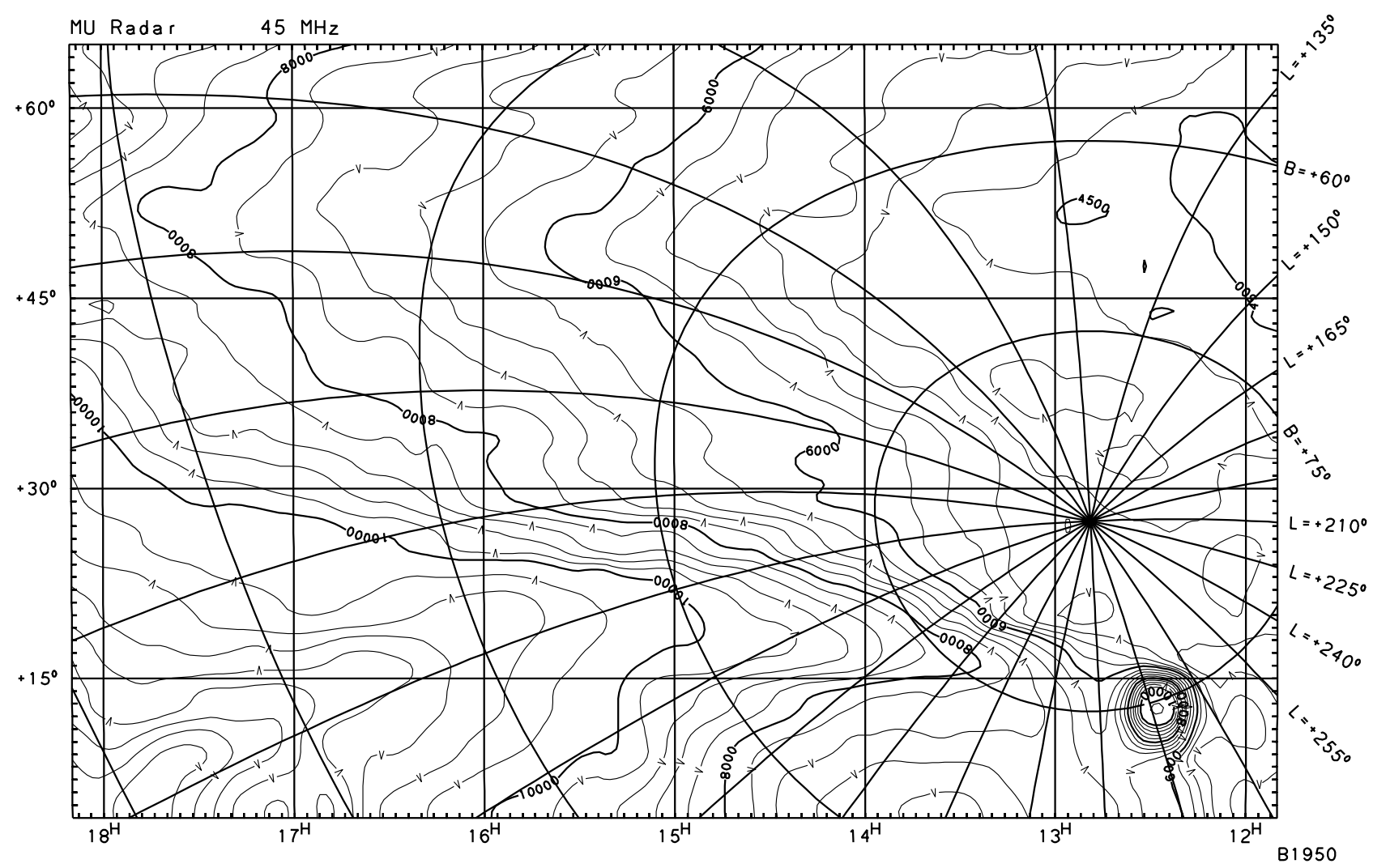

Fig. 1. continued

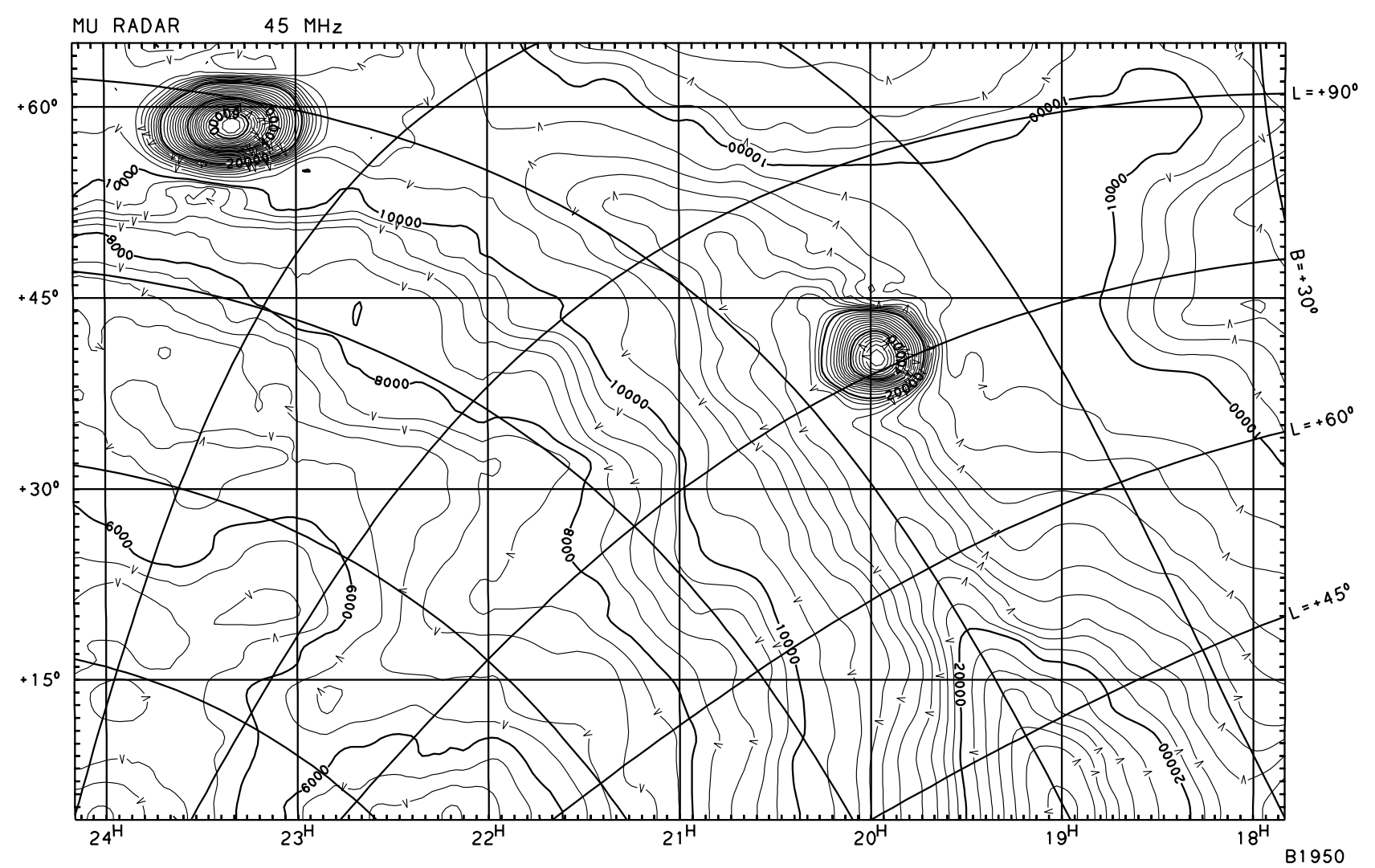

Fig. 1. continued 


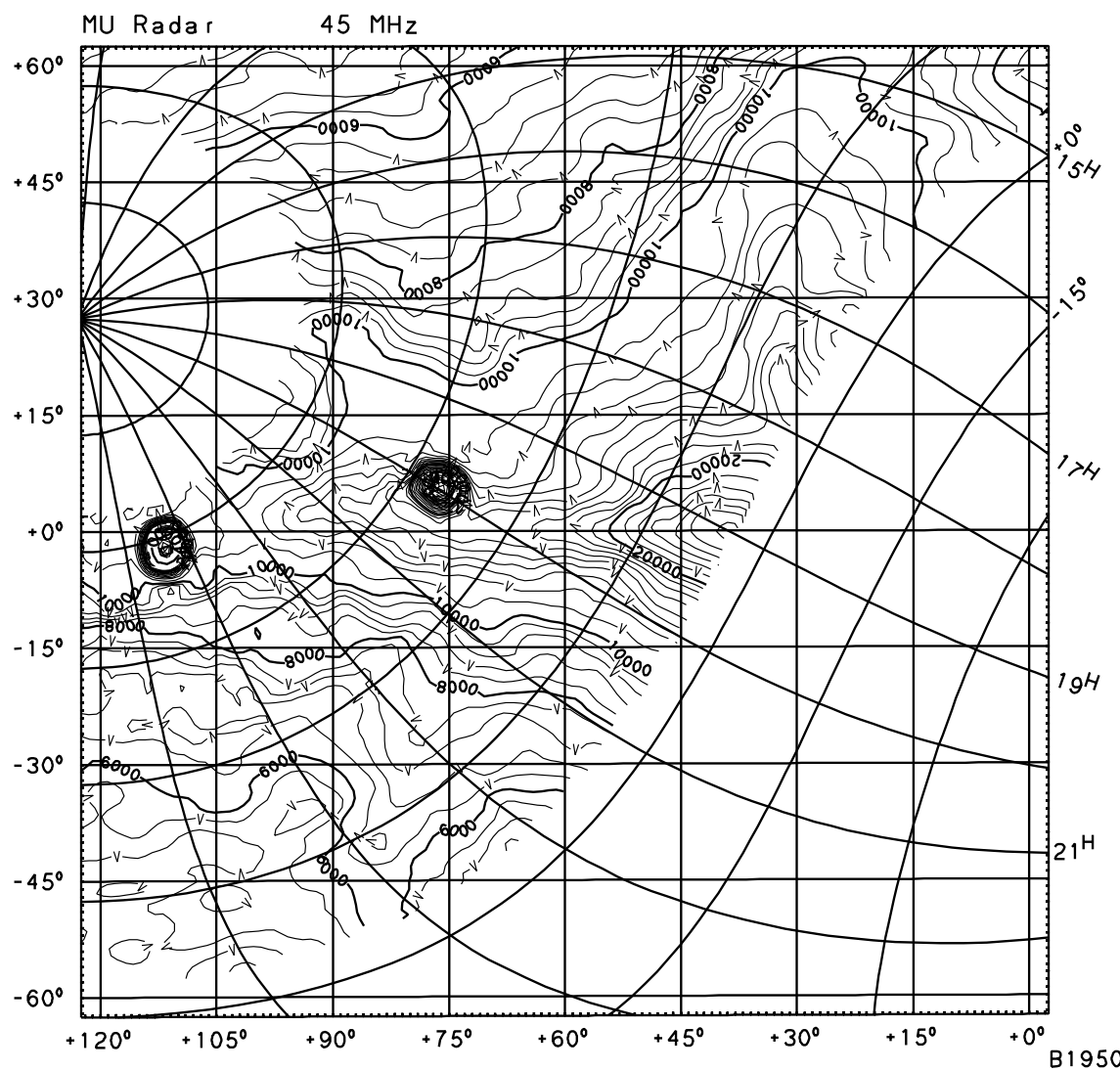

Fig. 2. Set of maps presented in galactic coordinates. The contour levels are the same as those in Fig. 1 (see text). Arrows on contour lines point towards decreasing temperatures

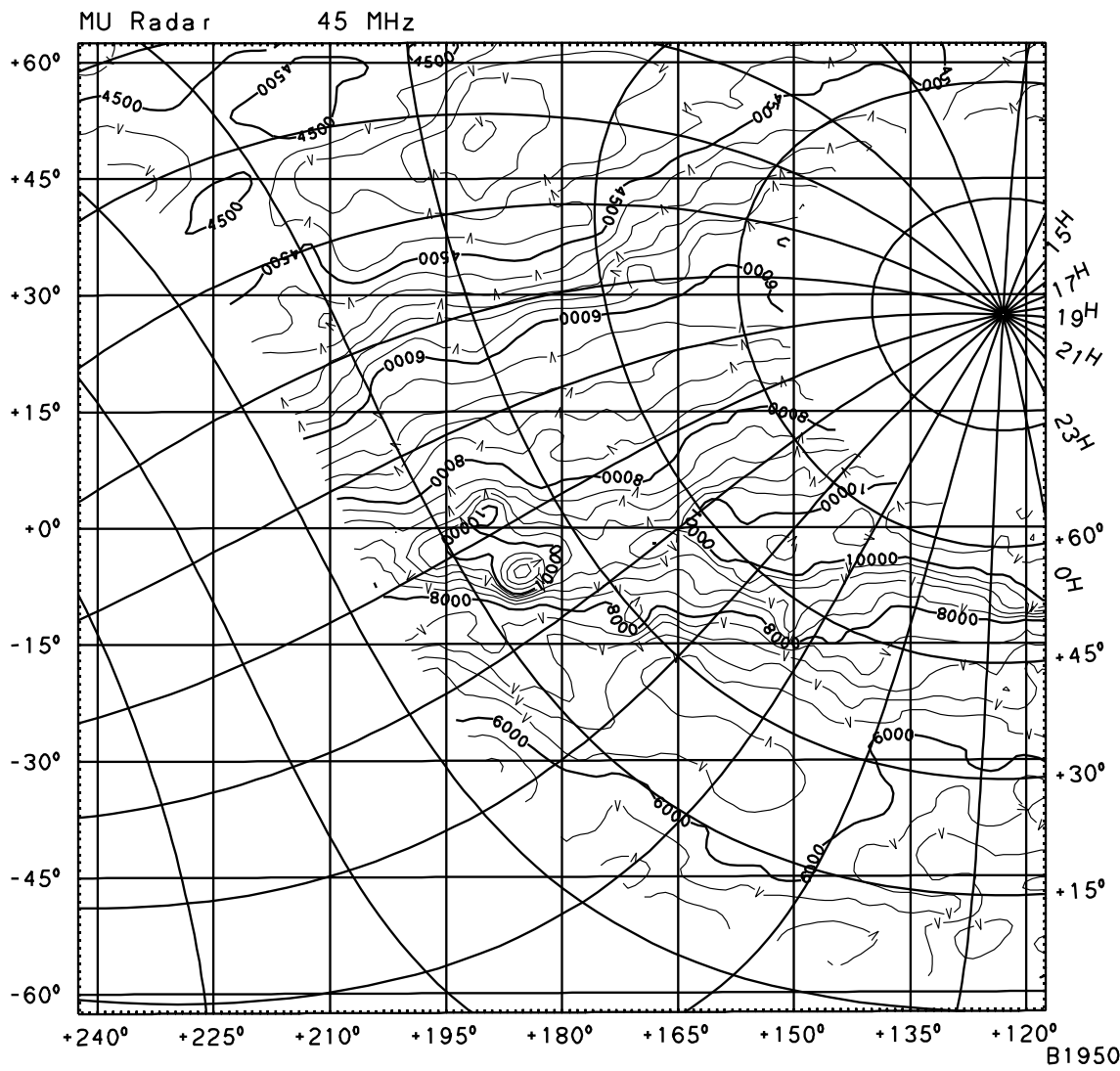

Fig. 2. continued 


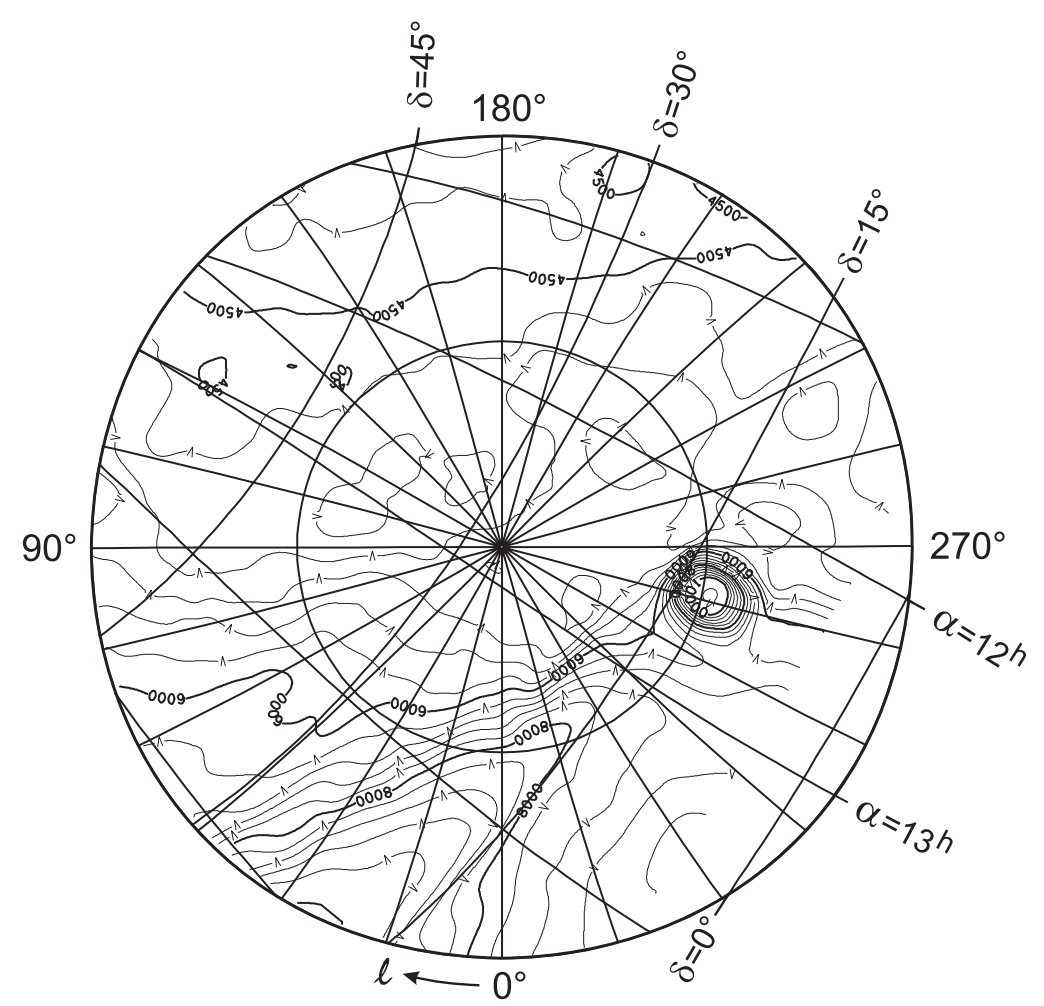

Fig. 2. continued

Next, the data acquisition rate of about one point every $10 \mathrm{~s}$, was changed to one data point per minute in right ascension in order to make it the same as in the Maipu data. Each data point was centered at the $00 \mathrm{~s}$ of each minute and it represents the integration of five or six original data points. The profiles obtained were calibrated against the scan at 4.85 , obtained from the Maipu survey. This 4.85 profile was selected because it was the overlapped position with best data in both surveys.

A plot of temperature from the Maipu Survey versus the relative intensity from Shigaraki data, for every point of a profile at a given declination, is defined as a T-T plot in what follows. By means of an iterative T-T plot analysis the northern relative intensity profile at 4.85 was calibrated to the corresponding temperature profile from the $45 \mathrm{MHz}$ southern survey. Even though the overall correlation was good (about 0.998 ), the 4.85 northern profile thus calibrated, showed differences along the day with respect to the 4.85 calibrator profile of the Southern Survey. We have attributed these differences to temperature gain variations of the MU radar receiver. To correct for these fluctuations we produced a curve formed by ratios of corresponding points in RA, in both profiles. Next we fitted a polynomial to this curve in order to have a continuous and smooth curve of ratios (factors). This curve of factors was applied to the calibrated profile and a new T-T plot analysis was done with the profile thus modified. This is an iterative process that continued until the curve of factors was practically flat at 1 . The convergence was fast and no more than three iterations were necessary. Then the final curve of factors, resulting from the product of the intermediate curves of factors, and the parameters derived from the $\mathrm{T}-\mathrm{T}$ plots were applied to the rest of the northern profiles. Since the measured temperature is an average of the brightness distribution within the beam, and independent of the antenna effective area, we did not apply any $Z$-dependent correction. This process was applied separately to each of the data sets. We have assumed that, in each of the declination positions of the stepping beam, the gain of the system remained the same.

Although the original northern sky data were taken at $46.5 \mathrm{MHz}$, the calibration process just described brings the data to $45 \mathrm{MHz}$. The basic assumption in this process is that at a given declination the shape of the profile is the same at both frequencies. This seems reasonable since the frequencies are very close.

In combining the 1988 and the 1998 data, the two coverages have been added by using an algorithm similar to PLAIT (Emerson \& Gräve 1988), with a double weight for the 1988 data. A further quality increase has been achieved by applying the so-called method of unsharp masking (Sofue \& Reich 1979).

\section{Presentation of the data}

The survey is presented in two sets of maps, i.e., in equatorial and galactic coordinates. In equatorial coordinates (Epoch 1950) the survey consists of 4 maps (Fig. 1), each 


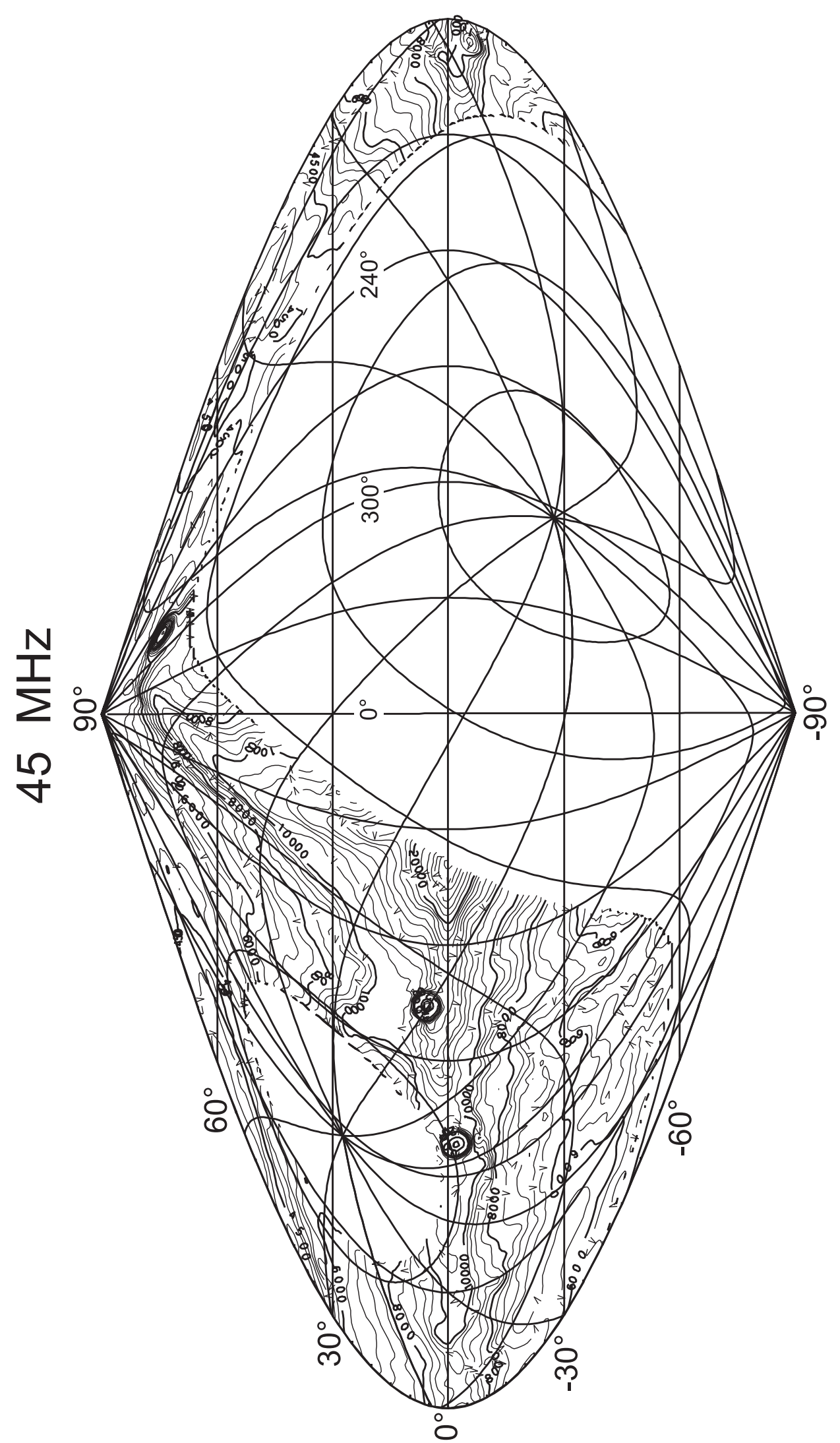

Fig. 3. Map presented in galactic coordinates in equal-area projection 


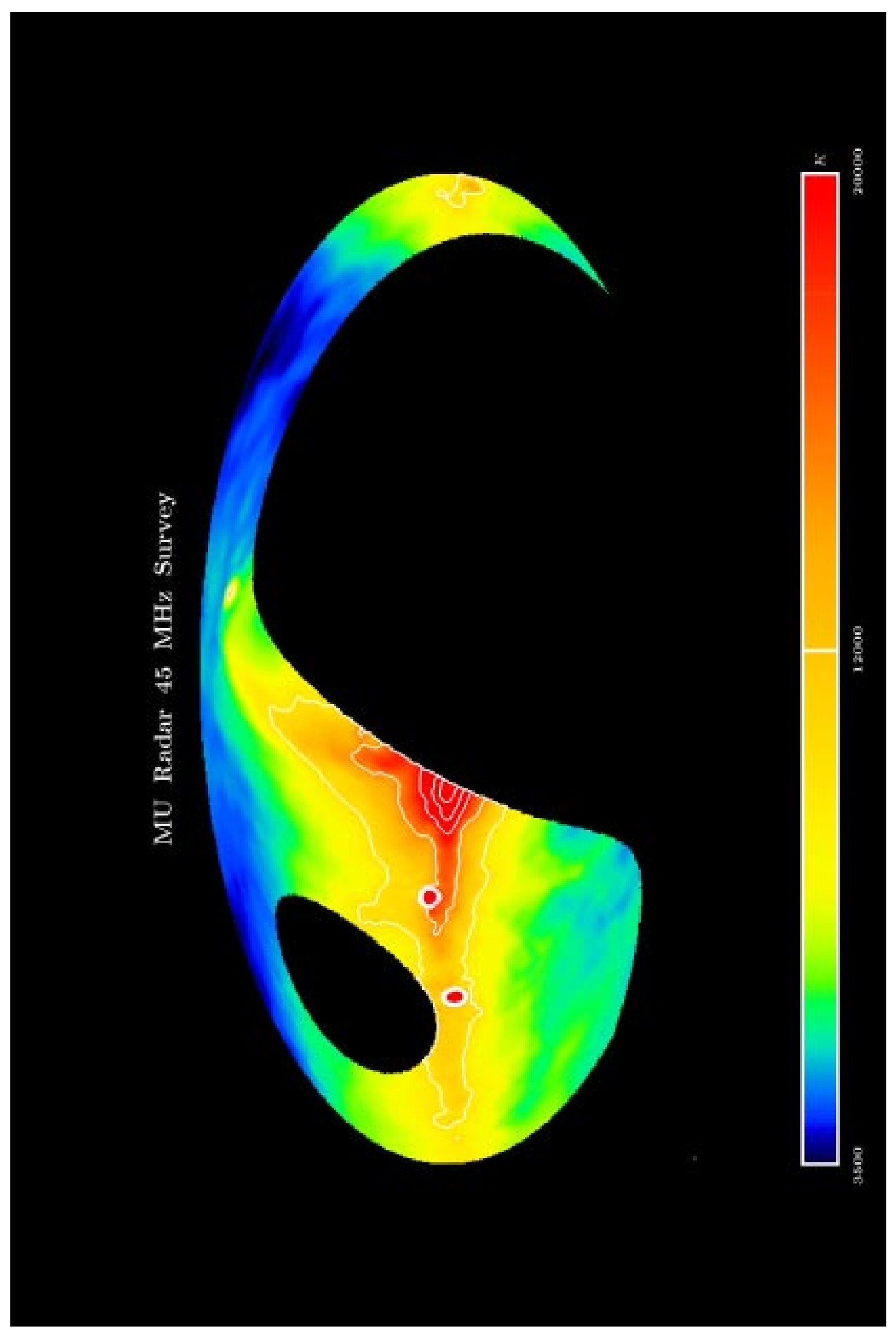

Fig. 4. Colour coded Aitoff projection map of the $45-\mathrm{MHz}$ survey in galactic coordinates. The code is shown in the figure. The contours begin at $10000 \mathrm{~K}$ and continue in steps of $5000 \mathrm{~K}$ 
Table 3. Low-frequency surveys below $100 \mathrm{MHz}$ in the northern hemisphere

\begin{tabular}{lrlll}
\hline $\begin{array}{l}\nu \\
(\mathrm{MHz})\end{array}$ & $\begin{array}{rll}\text { Coverage }(\delta) \\
\left({ }^{\circ}\right)\end{array}$ & $\begin{array}{l}\text { Resolution }(\alpha, \delta) \\
\left({ }^{\circ}\right)\end{array}$ & Instrument & Reference \\
\hline 81 & $-28-+82$ & $2 \times 15$ & Cylindrical paraboloid & Baldwin (1955) \\
45 & $+5.0-+65$ & $3.6 \times 3.6 \sec Z$ & Filled circular array & This work \\
38 & $-25-+70$ & 8 & Dish & Milogradov-Turin \& Smith (1973) \\
38 & $-15-+90$ & 0.75 & T-array & Williams et al. (1966) \\
38 & $-20-+70$ & $\sim 2.5$ & Moving-T & Blythe (1957) \\
34.5 & $-36-+64$ & $26^{\prime} \times 42^{\prime} \sec Z$ & T-array & Dwarakanath \& Udaya Shankar (1990) \\
22 & $-28-+80$ & $1.1 \times 1.7 \sec Z$ & T-array & Roger et al. (1999) \\
10 & $-5.0-+71$ & 2 & T-array & Caswell (1976) \\
\hline
\end{tabular}

covering $+4^{\circ} \leq \delta \leq+65^{\circ}$ and 6 hours in right ascension. In addition to a grid of equatorial coordinates, a grid of galactic coordinates is superposed in each map. In galactic coordinates the survey is shown in 3 maps (Fig. 2). A grid of equatorial coordinates (Epoch 1950) is superposed on the maps. Contour lines correspond to brightness temperature. The contour lines are drawn in $250 \mathrm{~K}$ steps up to $6000 \mathrm{~K}$, labelled every $1500 \mathrm{~K}$; from $6000 \mathrm{~K}$ to $10000 \mathrm{~K}$ in $400 \mathrm{~K}$ steps, labelled every $2000 \mathrm{~K}$; from $10000 \mathrm{~K}$ to $20000 \mathrm{~K}$ in $1000 \mathrm{~K}$ steps, labelled every $10000 \mathrm{~K}$; from $20000 \mathrm{~K}$ to $66000 \mathrm{~K}$ in $2000 \mathrm{~K}$ steps, labelled every $20000 \mathrm{~K}$. Arrows on contour lines point towards decreasing temperature. Figure 3 represents the survey in equal-area projection of galactic coordinates. Figure 4 shows a colour coded map of the whole survey in an Aitoff projection of galactic coordinates.

\section{Discussion}

In Table 3 we show the available low-frequency continuum surveys below $100 \mathrm{MHz}$ covering a relatively large area of the northern hemisphere. Milogradov-Turin \& Smith (1973) used the Jodrell Bank $76 \mathrm{~m}$ telescope for their survey at $38 \mathrm{MHz}$, which gives less than one-half of our resolution. Baldwin (1955) using a cylindrical paraboloid at $81 \mathrm{MHz}$ obtained a survey with even lower resolution. The synthesis surveys are intended to delineate small-scale features, and they are generally not reliable to study large scale structures. The surveys by Williams et al. (1966), by Caswell (1976) and by Blythe (1957) do not cover 24 hours of right ascension, while the maps by Dwarakanath \& Udaya Shankar (1990) are seriously contaminated by side lobe response to strong sources.

We believe that the calibration of the data presented here by using the $45-\mathrm{MHz}$ data from the Maipu survey was successful and that the estimated error is less than $15 \%$. This survey shows well the global features so far known, for instance, the north polar spur, the temperature minimum region around $\alpha=9^{\mathrm{h}} 30^{\mathrm{m}}, \delta=35^{\circ}$. Some discrete emission and absorption features are seen and their positions are in good agreement with those in the $22 \mathrm{MHz}$ map of Roger et al. (1999).
A frequency around $45 \mathrm{MHz}$ is not too high to be contaminated with thermal emission, nor too low to be affected by thermal free-free absorption (Alvarez et al. 1987). We therefore believe that the $45 \mathrm{MHz}$ survey represents almost pure synchrotron emission. Comparing this survey with others in different ranges of the electromagnetic spectrum will yield fruitful results. The Galaxy is a mixture of matter, radiation and magnetic fields that interact in complex ways. It is therefore not unexpected to find a correlation between some of the phases of the interestellar medium. For example, a tight linear correlation has been well established in spiral galaxies, between the logarithm of the far infrared (FIR) and the logarithm of the nonthermal radio continuum (NTRC) (e.g. Fitt et al. 1988; Helou et al. 1985; de Jong et al. 1985). Also a correlation between the NTRC and the CO line emission has been found in galaxies though it has not been as profusely investigated as the FIR case (Israel \& Rowan-Robinson 1984; Adler et al. 1991).

It is of interest to compare the low-frequency radio map with sensitive $\mathrm{H} \alpha$ surveys (e.g. Reynolds 1998) which indicate the distribution of the ionized hydrogen. At high galactic latitude, where optical extinction is small, the intensity of the $\mathrm{H} \alpha$ line is directly proportional to the emission measure, therefore also to the free-free opacity along the line of sight.

One of the future uses of the present survey, on which we are already working, is that it can be combined with the Maipu survey in order to get a map of the whole sky (except $4.7 \%$ of it around the north celestial pole). This will be a meaningful map since the northern and southern surveys have been made with practically the same angular resolution and frequencies. The only published all-sky map that gets close to meeting these conditions is that by Haslam et al. (1982) at $408 \mathrm{MHz}$. In the near future a $1420 \mathrm{MHz}$ all-sky map will be obtained with the northern data from Reich (1982) and Reich \& Reich (1986), and with the southern data from Testori et al. (in preparation). The combination of these unique all-sky surveys will allow us to obtain the spectral index of the radio background and its variation across the sky reflecting the energy distribution of the radiating relativistic electrons. 
Acknowledgements. The MU radar belongs to and is operated by the Radio Atmospheric Science Center, Kyoto University. We thank Dr. T. Sato, Dr. M. Yamamoto, and Dr. H. Hashiguchi for many suggestions about our observations. We also thank Dr. S. Maeda and Dr. Y. Muraoka for their contribution in the data processing. The assistance of F. Olmos in the data reduction is highly appreciated. We are specially grateful to Prof. R. Wielebinski for his continuous interest in and support of this project. We thank Prof. T.D. Carr for pointing out that we were doing similar work in both hemispheres. We appreciate helpful comments from Dr. W. Reich and Dr. R. Duncan. J.M, H.A., J.A., and K.M. acknowledge support from FONDECYT-Chile grant 8970017.

\section{References}

Adler D.S., Allen R.J., Lo K.Y., 1991, ApJ 382, 475

Alvarez H., Aparici J., May J., Olmos F., 1997, A\&AS 124, 315

Alvarez H., Aparici J., May J., 1987, A\&A 176, 25

Baldwin J.E., 1955, MNRAS 115, 684

Blythe J.H., 1957, MNRAS 117, 655

Caswell J.L., 1976, MNRAS 177, 601

de Jong T., Klein U., Wielebinski R., Wunderlich E., 1985, A\&A 147, L6

Dwarakanath K.S., Udaya Shankar N., 1990, JA\&A 11, 323
Emerson D.T., Gräve R., 1988, A\&A 190, 353

Fitt A.J., Alexander P., Cox M.J., 1988, MNRAS 233, 907

Fukao S., Sato T., Tsuda T., Kato S., Wakasugi K., Makihira T., 1985a, Radio Sci. 20, 1155

Fukao S., Tsuda T., Sato T., Kato S., Wakasugi K., Makihira T., 1985b, Radio Sci. 20, 1169

Haslam C.G.T., 1974, A\&AS 15, 333

Haslam C.G.T., Salter C.J., Stoffel H., Wilson W.E., 1982, A\&AS 47, 1

Helou G., Soifer B.T., Rowan-Robinson M., 1985, ApJ 298, L7

Israel F.P., Rowan-Robinson M., 1984, ApJ 283, 81

Milogradov-Turin J., Smith F.G., 1973, MNRAS 161, 269

Reich P., 1986, Ph.D. Thesis. University of Bonn

Reich P., Reich W., 1986, A\&AS 63, 205

Reich W., 1982, A\&AS 48, 219

Reynolds R.J., Tufte S.L., Haffner L.M., Jaehnig K., Percival J.W., 1998, Publ. Astron. Soc. Aust. 15, 14

Roger R.S., Costain C.H., Landecker T.L., Swerdlyk C.M., 1999, A\&A 137, 7

Salter C.J., Brown R.L., 1988, in: Verschuur G.L. and Kellermann K.I. (eds.) Galactic and Extragalactic Radio Astronomy (second edition). Springer-Verlag, New York, p. 1

Sofue Y., Reich W., 1979, A\&AS 38, 251

Testori J.C., Reich P., et al., A\&AS (in preparation)

Williams P.J.S., Kenderdine S., Baldwin J.E., 1966, Mem. R. Astron. Soc. 70, 53 